\title{
Flexible PCL tube scaffolds by winding of micro-extruded filaments
}

\author{
K. Ragaert \& L. Cardon \\ CPMT group, Faculty of Applied Engineering Sciences, University College Ghent, \\ Voskenslaan 362, B-9000 Ghent, Belgium and Department of Materials Science \& Engineering, Ghent \\ University, Technologiepark 903, 9052 Ghent, Belgium
}

J. Degrieck

Department of Materials Science \& Engineering, Ghent University, Technologiepark 903, B-9052 Ghent, Belgium

ABSTRACT: An important requirement for tissue engineering scaffolds is matching of the functional mechanical properties to their natural tissue counterpart. Specifically for arteries this comprises the elastic response of the vessel wall to blood pressure. Human aorta has a low elastic modulus when compared to some FDA-approved synthetic polymer materials frequently used in tissue engineering. The current research endeavours to expand the existing production technology of 3D plotting to winding of micro-extruded filaments in order to obtain flexible polymer tubes with continuous fibre. Tube scaffolds are manufactured by conventional 3D plotting and by winding. Their structure and quasi-static mechanical properties are evaluated and compared to human aorta. Winded tubes are found to be far more suitable for application as a blood vessel scaffold than their 3D plotted counterparts.

\section{INTRODUCTION}

The creation of a functional tissue engineered blood vessel (TEBV) for arteries with lumen diameters of $6 \mathrm{~mm}$ and smaller is widely researched, yet has met with little clinical success up to date [1-3]. Next to biocompatibility and biodegradability, an important aspect of TEBV properties is elasticmechanical compliance with its natural tissue counterpart. The TEBV must be strong enough to withstand rupture at elevated blood pressures (functionality of collagen) and at the same time be able to follow the elastic movement of a natural artery (functionality of elastin). Where synthetic scaffolds are concerned, it has proven difficult to obtain both qualities in the same part. Often, ultimate mechanical properties are more than sufficient but flexible behaviour under smaller loads cannot be emulated.

In the current research, we investigate the mechanical suitability of polymeric tubes for use as TEBV. Poly- $\varepsilon$-caprolactone (PCL) is a suitable material for applications that require more pliant polymer materials. Both at room and body temperature, PCL is above its glass transition and quite flexible when compared to FDA-approved aliphatic polyesters like poly-(lactid acid) PLA of poly-(glycolic acid) PGA [4]. PCL has equally shown promise during in vivo experiments of an electrospun TEBV [5].

PCL tubes are created by both conventional 3D plotting and filament winding. Their static mechanical properties are assayed by tensile testing of ring segments, an oft-used for mechanical assay of TEBV
[6-8]. The resulting circumferential modulus values are compared to those found in literature for human aorta.

Some older reports describe measurements of mechanical properties for the human aorta, most of which are based on the principles of pressurediameter experiments [9-11]. For the modulus in the circumferential direction, values of $0.75 \mathrm{MPa}$ to 1.7 MPa are noted for thoracic and abdominal aorta segments [10-13].

Whilst several groups have reported on the creation of PCL tube scaffolds with randomly oriented fibre through electrospinning [14-16], to our knowledge this manuscript presents the first results on adapting the 3D plotting technique for winding of the extruded filament into PCL tubes with continuous fibre.

\section{METHODS \& MATERIALS}

\subsection{Materials}

Thermoplastic polymer material used is granulated PCL CAPA 6500 from Perstorp with number averaged molecular mass of $50000 \mathrm{Da}$ and a melt temperature of $60^{\circ} \mathrm{C}$ [17].

\subsection{BioScaffolder apparatus}

All tube scaffolds were produced on a BioScaffolder apparatus (SysEng). The manufacturing technique of the BioScaffolder process has been previously de- 
scribed in literature [18, 19]. In brief, polymer granulate is extruded by a mobile dispense head into fine filaments (order $100-800 \mu \mathrm{m}$ ). These filaments are deposited on a plotting table layer by layer, building up a 3D part in an additive fashion.

PCL was processed at $110^{\circ} \mathrm{C}$ with a thermoplastics dispense head. A $28 \mathrm{~g}$ needle was used, producing extruded filaments $180 \mu \mathrm{m}$ in diameter. Spindle speed of the extrusion screw was 22rpm. Parameters specific to each tube series are described below.

\section{$2.33 D$ plotted tube scaffolds}

Three series of tube scaffolds were created by conventional 3D plotting with inner diameter $\mathrm{d}_{\text {in }}=$ $6 \mathrm{~mm}$, wall thickness $\mathrm{t}=0.5 \mathrm{~mm}$ and tube height $\mathrm{h}=$ $5 \mathrm{~mm}$. For the first series the orientation shift between two consequent layers was $45^{\circ}$, for the second it was $30^{\circ}$ and for the third $15^{\circ}$. These series are respectively labelled "3Dplotted-45", "3Dplotted-30" and "3Dplotted-15". Most important processing parameters were set as follows: dispense head XY feed $80 \mathrm{~mm} / \mathrm{min}$, dispense pressure 5bar, layer thickness $160 \mu \mathrm{m}$ and strand distance $324 \mu \mathrm{m}$.

\subsection{Winded tube scaffolds}

Tubes with continuous fibre were realized by the implementation of a rotational axis on the BioScaffolder apparatus, onto which the extruded filaments are winded (shaft speed $=3 \mathrm{rpm}$ ). This is illustrated in figure 1 . Tube scaffolds were designed with $d_{\text {in }}=$ $6 \mathrm{~mm}, \mathrm{t}=0.5 \mathrm{~mm}$ and $\mathrm{h}=9-10 \mathrm{~mm}$. Different filament winding angles were accomplished by adapting the dispense head feed F. Three series of winded tube scaffolds were created on a rotating axis of $6 \mathrm{~mm}$ with the following winding angles: $10^{\circ}, 45^{\circ}$ and a combination of both. These series are respectively labelled "winded-10", "winded-45" and "winded-C".

\subsection{Scaffold inspection}

Visual evaluation of scaffolds was performed with a Keyence VHX 500 microscope. Scaffold height and wall thickness was assessed with a digital calliper.

\subsection{Tensile testing}

Evaluation of mechanical properties was done on a Instron 5565 tensile testing apparatus, with a load cell of $10 \mathrm{~N}$. Tubes were mounted over a double pin setup, which is shown in figure 2. Start length was calculated as the circumference of the tube and strain was determined in relation to this length. Tubes were loaded at rate of $10 \mathrm{~mm} / \mathrm{min}$ to a maximum load of
$10 \mathrm{~N}$. E-modulus was determined as the slope of the linear ascending part of the stress-strain curve, meaning $\sigma \geq 1 \mathrm{MPa}$ for 3D plotted tubes and $\sigma \geq 0.4$ $\mathrm{MPa}$ for the winded tubes. Contrary to natural tissue, it was not necessary to precondition the tubes prior to measurement, as the elastic behaviour does not vary with increasing load cycle. Modulus was determined for 3 tubes per production series and results averaged.

\section{RESULTS}

\subsection{Manufactured tube scaffolds}

An overview of the dimensions of the fabricated tubes is given in table 1. All dimensions remain within an acceptable standard deviation of less than $1 \%$ for height and less than $5 \%$ for wall thickness, which speaks well for the reproducibility of the manufacturing technique.

Table 1: dimensions of fabricated tubes

\begin{tabular}{l|cccccc}
\multirow{2}{*}{ tube type } & $\begin{array}{c}\text { dimen- } \\
\text { sion }\end{array}$ & tube1 & tube2 & tube3 & mean & stdev \\
\cline { 2 - 7 } & $\mathrm{h}[\mathrm{mm}]$ & 5,01 & 5,00 & 5,03 & 5,01 & 0,02 \\
& $\mathrm{t}[\mathrm{mm}]$ & 0,52 & 0,51 & 0,54 & 0,52 & 0,02 \\
\hline 3Dplotted-30 & $\mathrm{h}[\mathrm{mm}]$ & 5,02 & 5,04 & 5,01 & 5,02 & 0,02 \\
& $\mathrm{t}[\mathrm{mm}]$ & 0,52 & 0,51 & 0,54 & 0,52 & 0,02 \\
\hline 3Dplotted-15 & $\mathrm{h}[\mathrm{mm}]$ & 5,01 & 5,01 & 5,00 & 5,01 & 0,01 \\
& $\mathrm{t}[\mathrm{mm}]$ & 0,53 & 0,54 & 0,52 & 0,53 & 0,01 \\
\hline Winded-10 & $\mathrm{h} \mathrm{[mm]}$ & 9,53 & 9,63 & 9,54 & 9,57 & 0,06 \\
& $\mathrm{t}[\mathrm{mm}]$ & 0,52 & 0,48 & 0,51 & 0,50 & 0,02 \\
\hline Winded-45 & $\mathrm{h}[\mathrm{mm}]$ & 9,08 & 8,97 & 9,04 & 9,03 & 0,06 \\
& $\mathrm{t}[\mathrm{mm}]$ & 0,54 & 0,53 & 0,52 & 0,53 & 0,01 \\
\hline Winded-C & $\mathrm{h}[\mathrm{mm}]$ & 9,65 & 9,70 & 9,71 & 9,69 & 0,03 \\
& $\mathrm{t}[\mathrm{mm}]$ & 0,52 & 0,48 & 0,51 & 0,50 & 0,02 \\
& & & & &
\end{tabular}

Figure 3 shows the resulting geometry of a 3D plotted tube with the $45^{\circ}$ stacking sequence. To obtain a flexible tube scaffold, there are several disadvantages to structures created by conventional 3D plotting.

For one, the layer-wise production method effectively limits polymer filament distribution to the individual layers; there is no continuous filament in the longitudinal/axial direction of the tube. This may cause delamination of the scaffold layers when axially loading the tube and could be the case for suturing the TEBV into position or mounting it on a bioreactor for conditioning. Moreover, it leads to expect a high stiffness in the radial \& circumferential direction which is not desirable for arterial replacements.

Secondly, the dispense head is forced to manoeuvre back and forth in the thin section of the tube 
wall, which leads to a very dense structure with little to no remaining porosity in the scaffold wall.

Finally, in every layer there are two sections of the tube wall in which the deposited filament does not meander back and forth within the wall thickness, but draws a straight line. The phenomenon is inherent to the postprocessor, which attempts to deposit as much continuous filament as possible in the direction aligned to the current layer orientation. This straight line is repositioned in each subsequent layer according to the stacking angle. Hence, a $45^{\circ}$ stacked tube wall tube will display this anomaly every $4^{\text {th }}$ layer and within 4 longitudinal sections of the tube, whilst a $15^{\circ}$-stacked part will only exhibit it every $12^{\text {th }}$ layer for a single wall section, but also in 12 different locations. We summarize this phenomenon as follows:

$$
\mathrm{n}_{\mathrm{w}}=360 / \alpha
$$

with $\alpha$ the scaffold stacking angle and $\mathrm{n}_{\mathrm{w}}$ both the number of wall sections in the circumference where and every how many layers the weakness occurs within these sections.

Figure 4 shows the three types of winded scaffolds. The tube winded under low angle (winded10), not unlike the 3D plotted tubes, turns out to be a very dense and closed structure. The one winded under $45^{\circ}$ (winded-45) however, displays increased porosity in the tube wall. Finally, porosity in the tube with combined winding angles (winded-C) is governed by the density of the outer layer - winded under low angle - which will affect pore size and geometry.

All of the winded tubes present continuous polymer fibres over the entire length of the scaffold.

These winded tubes feel much more pliant to the touch than their 3D plotted counterparts, hinting at lower elastic modulus values.

\subsection{Tensile properties}

The results of the quasi-static tensile tests are summarized in table 2 and visualized in figure 5.

Table 2: experimental tensile modulus for tube scaffolds

\begin{tabular}{l|ccccc} 
tube type & $\begin{array}{c}\mathbf{E}_{\text {tube1 }} \\
{[\mathrm{MPa}]}\end{array}$ & $\begin{array}{c}\mathbf{E}_{\text {tube2 }} \\
{[\mathrm{MPa}]}\end{array}$ & $\begin{array}{c}\mathbf{E}_{\text {tube3 }} \\
{[\mathrm{MPa}]}\end{array}$ & $\begin{array}{c}\mathbf{E}_{\text {mean }} \\
{[\mathrm{MPa}]}\end{array}$ & $\begin{array}{c}\text { stdev } \\
{[\mathrm{MPa}]}\end{array}$ \\
\hline 3Dplotted-45 & 18,05 & 16,38 & 19,36 & 17,93 & 1,49 \\
3Dplotted-30 & 17,11 & 19,5 & 21,17 & 19,26 & 2,04 \\
3Dplotted-15 & 22,68 & 23,63 & 25,16 & 23,82 & 1,25 \\
Winded-10 & 12,11 & 13,377 & 12,307 & 12,60 & 0,68 \\
Winded-45 & 4,238 & 4,587 & 4,75 & 4,53 & 0,26 \\
Winded-C & 7,5718 & 8,011 & 7,618 & 7,73 & 0,24
\end{tabular}

The 3D plotted tubes exhibit stiffness values between $16 \mathrm{MPa}$ and $26 \mathrm{MPa}$, which is about 200 times the earlier reported moduli for arterial tissue [10-13]. Stiffness tends to rise with diminishing stacking angle. As shown in figure 6, visual inspection of these scaffolds after testing reveals a peculiar mode of straining. A plastic deformation is observed within a single section of the tube wall. One may note from figure 6-c that exactly every $\mathrm{n}_{\mathrm{w}}^{\text {th }}$ layer is broken, causing the tensile force to be redistributed over the remaining scaffold layers and further weakening the structure. This section corresponds to the locations of the straight-deposited filament discussed above, confirming them out as influential flaws in the wall structure.

For the winded scaffolds, remarkably lower modulus values are achieved. The open structure of the winded-45 series attains tensile modulus values of $4.53 \pm 0.26 \mathrm{MPa}$, less than a quarter of the stiffness of the 3D plotted tubes. Even the winded-10 series which is closest in structure to the dense plotted tubes, displays a distinctly reduced modulus of 12.60 $\pm 0.68 \mathrm{MPa}$. As could be expected, the winded-C series with combined winding angles shows values between those of its separate composing structures, with a modulus of $7,73 \pm 0.24 \mathrm{MPa}$.

There is less variation in modulus values between samples within the different winded series than within the 3D plotted series and the tubes did not display plastic deformation after tensile testing.

\section{DISCUSSION}

Compared to natural arteries, 3D plotted tube scaffolds for TEBV display a radial stiffness that is much too high, due to a very dense wall structure and the layer-wise assembly. They do not sport a continuous fibre throughout the tube height and the structural weakness induced by the periodical deviation from the meandering pathway within a layer causes them to partially fracture and then deform plastically during loading. It is possible to minimize this flaw by using smaller stacking angles, distributing the flaw along the tube circumference but this leads to overall stronger and stiffer tubes, exactly the property we are trying to downplay for use as functional TEBV. The lack of wall porosity will equally have a negative effect on nutrient transport and cell migration throughout the scaffold. Quite clearly, the 3D plotted tubes fabricated here are unfit for use as flexible TEBV scaffolds.

On the other hand, winding of extruded PCL filament offers auspicious results in term of both wall porosity and mechanical properties. A useful advantage of the winding technique which is evident from these simple experiments is the presence of a continuous fibre over the entire tube length, resulting in a more homogeneous straining behaviour instead 
of local failure. Lack of aforementioned localized structural weakness also ensures smaller variance in stiffness results.

It is clear from visual inspection that the tubes are more porous than their plotted counterparts. Such porosity may be fine-tuned by playing with dispense head feed and speed of the rotational axis, working towards either larger or smaller pores. Both this porosity and the directional orientation of the polymer filaments leads to lower values for the circumferential modulus. The most pliant result was obtained for the winded-45 series, with an average modulus of 4,53 MPa. While this does not yet emulate the low stiffness values of the arterial tissue, it most certainly indicates that this adapted production technique is a step in the good direction for creating flexible tube scaffolds for TEBV with synthetic polymer materials.

When abstracting the tube scaffolds to a thinwalled pressure vessel, the following relation between circumferential tensile stress and equivalent internal fluid pressure may be made :

$$
\begin{aligned}
& \sigma_{\theta}=\left(p^{*} d_{\text {out }}\right) / t \\
& \sigma_{t, \theta}=\mathrm{F} /\left(2 * h^{*} \mathrm{t}\right) \\
& \sigma_{\theta}=\sigma_{\mathrm{t}, \theta} \Leftrightarrow \mathrm{p}=\mathrm{F} /\left(2 * \mathrm{~h}^{*} \mathrm{~d}_{\text {out }}\right)
\end{aligned}
$$

With $\sigma_{\theta}$ the circumferential stress component induced by a fluid pressure $p$ on a thin-walled tube with outer diameter $\mathrm{d}_{\text {out }}$ and wall thickness $\mathrm{t}$ and $\sigma_{t, \theta}$ the stress induced on two circumferential tube wall sections by the tensile testing with a load force $\mathrm{F}$ on a tube with height $\mathrm{h}$ and wall thickness $\mathrm{t}$.

As such, the onset values for the linear elastic behaviour and the determination of elastic modulus correspond to roughly $1100 \mathrm{mmHg}$ blood pressure for $3 \mathrm{D}$ plotted tubes and $430 \mathrm{mmHg}$ for the winded tubes. This high value sustains the earlier conclusion that the plotted tubes are not at all suited to mimicking the behaviour of natural arteries and that winded tubes show greater promise, even whilst they are still some way off from reaching the very flexible nature of the arterial tissue itself.

It would seem that two of the main different approaches for constructing a TEBV may be advancing towards one another. Synthetic polymers are generally too stiff, but adequate processing through filament winding or electrospinning on a rotating mandrel $[5,14-16]$ is able to produce more and more flexible structures. On the other hand, biological polymer-based endeavours like collagen-based scaffolds for TEBV - which display a much greater attractiveness to cells -, have proven to be too weak in terms of tensile properties [2, 20, 21] but researchers are able to gradually improve these properties, for example by mechanical preconditioning [21]. Perhaps hybrid scaffolds that combine a synthetic polymer backbone for strength with natural material for flexibility and cell interaction will turn out to be a feasible solution for this intricate challenge of creating a functional TEBV, both towards cell interactions and mechanical behaviour. Efforts have already been made in this direction, reporting tensile moduli of $3.8 \mathrm{MPa}$ for an electrospun $\mathrm{PCL} /$ collagen combined scaffold [22].

\section{CONCLUSIONS}

In this research, it was demonstrated that the winding method for tube scaffolds is not only viable but also renders distinctly more flexible products than with traditional 3D plotting. This makes them more suitable as a replacement for the very elastic natural arterial tissue, even though close approximation of the circumferential modulus of arteries was not yet achieved. Further research should delve into attaining these low elastic moduli by adapting filament sizes, processing parameters and winding angles.

\section{ACKNOWLEDGEMENTS}

The authors would like to thank Mustafa Erkoç for his help with the development of the tensile test setup, as well as Adrian Badia \& Jose Luis Moreno for their assistance with the scaffold production \& tensile testing.

This research was funded by Faculty of Applied Engineering Sciences at University College Ghent.

\section{REFERENCES}

1. Isenberg, B.C., C. Williams, and R.T. Tranquillo, Small-diameter artificial arteries engineered in vitro. Circulation Research, 2006. 98(1): p. 25-35.

2. Gong, Z.D. and L.E. Niklason, Blood vessels engineered from human cells. Trends in Cardiovascular Medicine, 2006. 16(5): p. 153-156.

3. Yow, K.H., et al., Tissue engineering of vascular conduits. British Journal of Surgery, 2006. 93(6): p. 652-661.

4. Van de Velde, K. and P. Kiekens, Biopolymers: overview of several properties and consequences on their applications. Polymer Testing, 2001. 21: p. 433-442.

5. Pektok, E., et al., Degradation and Healing Characteristics of Small-Diameter Poly(epsilon-Caprolactone) Vascular Grafts in the Rat Systemic Arterial Circulation. Circulation, 2008. 118(24): p. 2563-2570.

6. Assoul, N., et al., Mechanical properties of rat thoracic and abdominal aortas. Journal 
of Biomechanics, 2008. 41(10): p. 22272236.

7. Buttafoco, L., et al., Physical characterization of vascular grafts cultured in a bioreactor. Biomaterials, 2006. 27(11): p. 2380-2389.

8. Wille, J.J., E.L. Elson, and R.J. Okamoto, Cellular and matrix mechanics of bioartificial tissues during continuous cyclic stretch. Annals of Biomedical Engineering, 2006. 34(11): p. 1678-1690.

9. Bergel, H., The properties of Blood Vessels, in Biomechanics, Its Foundations and Objectives, Y.C. Fung, N. Ferrone, and M. Anliker, Editors. 1972, Prentice Hall: New Jersey.

10. Langewouters, G.J., K.H. Wesseling, and W.J.A. Goedhard, The Static Elastic Properties of 45 Human Thoracic and 20 Abdominal Aortas Invitro and the Parameters of a New Model. Journal of Biomechanics, 1984. 17(6): p. 425-435.

11. Silver, F.H., D.L. Christiansen, and C.M. Buntin, Mechanical-Properties of the Aorta a Review. Critical Reviews in Biomedical Engineering, 1989. 17(4): p. 323-358.

12. Learoyd, B.M. and M.G. Taylor, Alterations with age in viscoelastic properties of human arterial walls. Circulation Research, 1966. 18(3): p. 278-\&.

13. Drangova, M., et al., Elasticity and geometry measurements of vascular specimens using a high-resolution laboratory CT scanner. Physiological Measurement, 1993. 14(3): p. 277-290.

14. Murugan, R. and S. Ramakrishna, Design strategies of tissue engineering scaffolds with controlled fiber orientation. Tissue Engineering, 2007. 13(8): p. 1845-1866.

15. Stitzel, J., et al., Controlled fabrication of a biological vascular substitute. Biomaterials, 2006. 27(7): p. 1088-1094.

16. Vaz, C.M., et al., Design of scaffolds for blood vessel tissue engineering using a multi-layering electrospinning technique. Acta Biomaterialia, 2005. 1(5): p. 575-582.

17. Perstorp, Capa 6000 series data sheet, P.U. Ltd, Editor 2003.

18. Ragaert, K., et al., Machine design \& processing considerations for the $3 D$ plotting of thermoplastic scaffolds. Biofabrication, 2010. 2 (1).

19. Landers, R., et al., Fabrication of soft tissue engineering scaffolds by means of rapid prototyping techniques. Journal of Materials Science, 2002. 37(15): p. 3107-3116.

20. Kakisis, J.D., et al., Artificial blood vessel: The Holy Grail of peripheral vascular surgery. Journal of Vascular Surgery, 2005. 41(2): p. 349-354.

21. Seliktar, D., et al., Dynamic mechanical conditioning of collagen-gel blood vessel constructs induces remodeling in vitro.
Annals of Biomedical Engineering, 2000. 28(4): p. 351-362.

22. Lee, S.J., et al., Development of a composite vascular scaffolding system that withstands physiological vascular conditions. Biomaterials, 2008. 29(19): p. 2891-2898. 


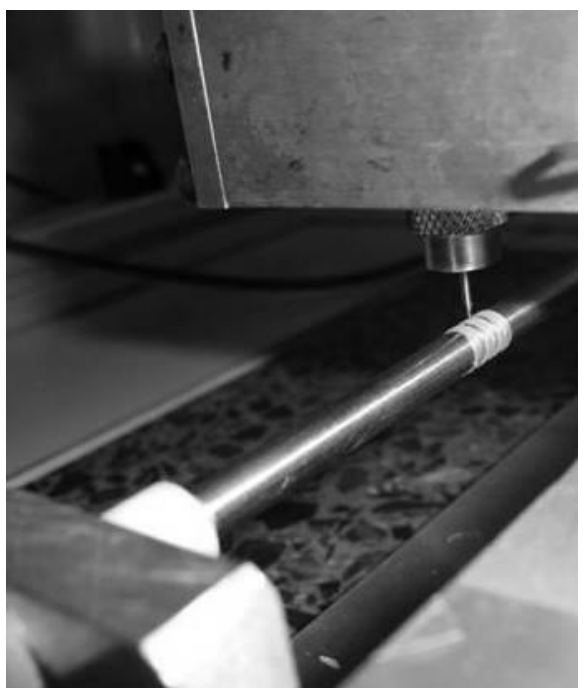

Figure 1: winding of tube scaffolds on BioScaffolder apparatus

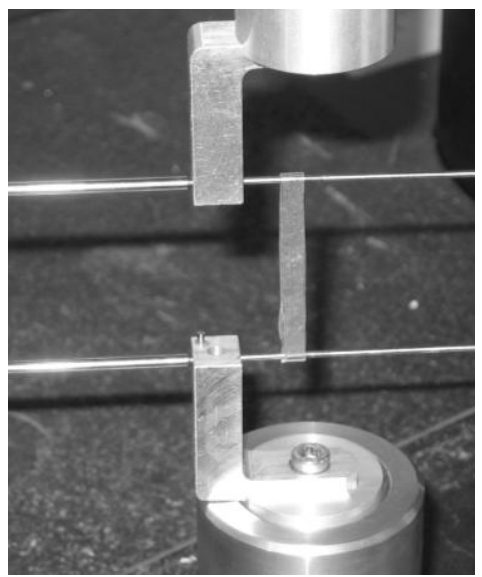

Figure 2: tensile test setup with pins
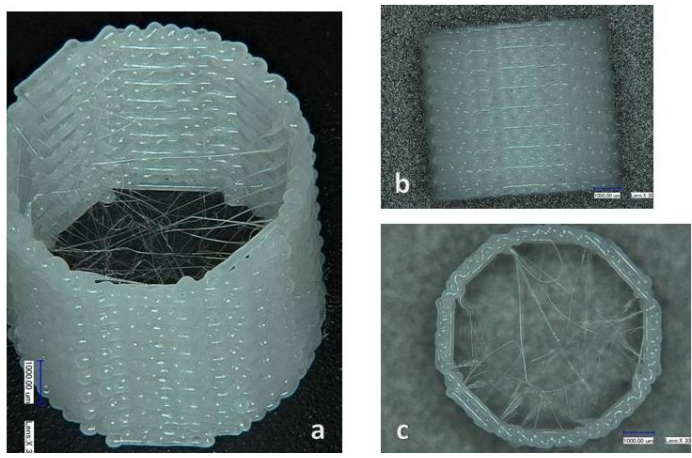

Figure 3: 3D plotted tube of $45^{\circ}$ type. (a) isometric view, (b) side view and (c) top view. All scale bars are $1 \mathrm{~mm}$.

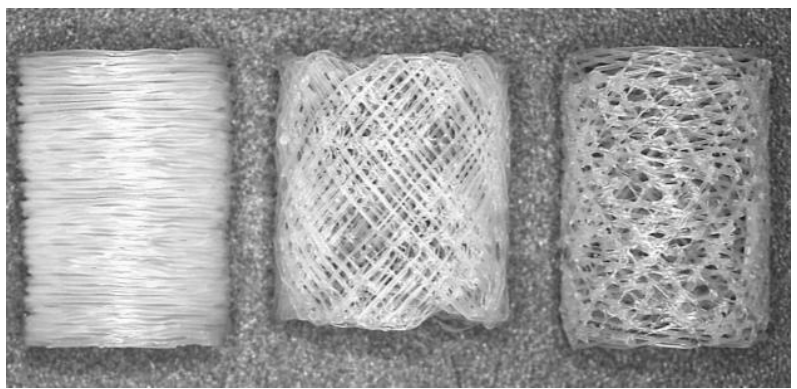

Figure 4: from left to right: tubes from series winded-10, winded-45 and winded-C.

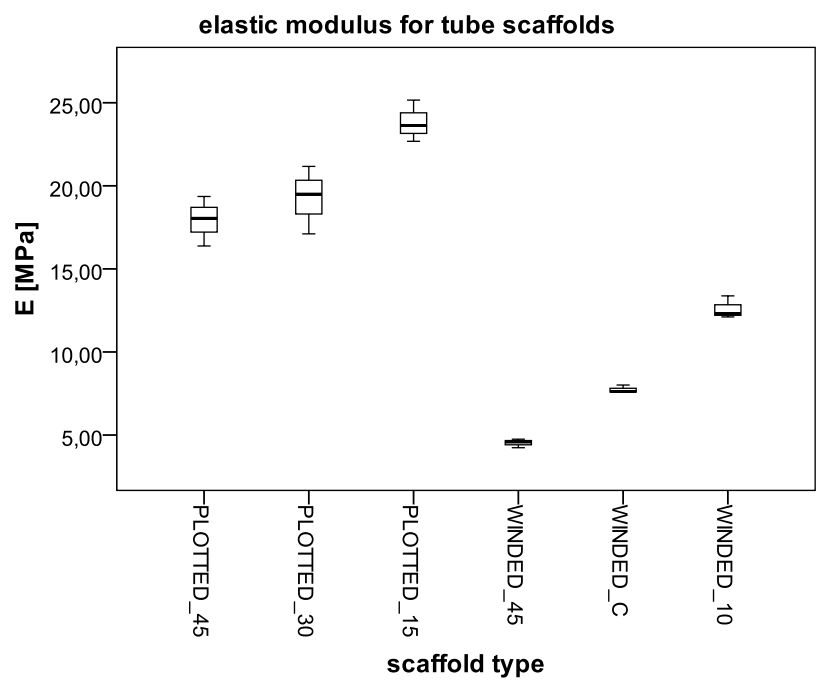

Figure 5: static elastic modulus values for all scaffold types
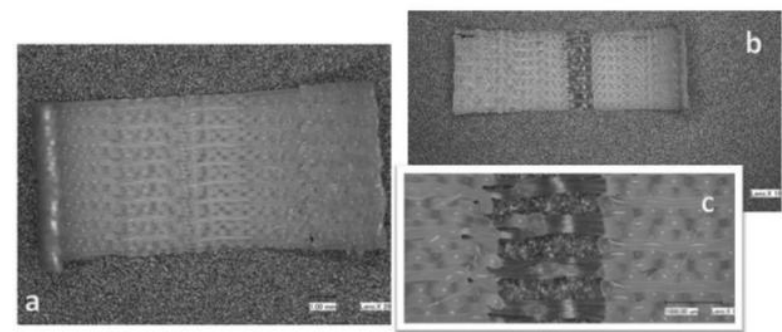

Figure 6: evaluation of $3 D$ plotted tube with $45^{\circ}$ stacking sequence. (a) cut-open tube before tensile loading. (b) cut-open tube after loading and (c) enlargement of strained region in tube. All scale bars are $1 \mathrm{~mm}$. 